\title{
Truckers Drive Their Own Assessment for Obstructive Sleep Apnea: A Collaborative Approach to Online Self-Assessment for Obstructive Sleep Apnea
}

\author{
Ben Smith, M.S. ${ }^{1}$; Barbara A. Phillips, M.D., M.S.P.H. ${ }^{2}$ \\ ${ }^{1}$ Department of Computer Science, North Carolina State University, Raleigh, NC; ${ }^{2}$ Division of Pulmonary, Critical Care and Sleep \\ Medicine, University of Kentucky College of Medicine, Lexington, KY
}

Background: Commercial motor vehicle drivers are at an increased risk for obstructive sleep apnea (OSA). The Federal Motor Carrier Safety Administration (FMCSA) Medical Review Board has recommended that commercial motor vehicle drivers undergo testing for OSA if they have a positive Berlin Questionnaire or a BMI $\geq 30 \mathrm{~kg} / \mathrm{m}^{2}$. We developed an online screening tool based on the Berlin Questionnaire for anonymous use by commercial drivers to assess their risk of OSA prior to their required FMCSA physicals.

Methods: We based the survey on the Berlin Sleep Questionnaire. The survey was hosted on the Truckers for a Cause Chapter of Alert Well and Keeping Energetic of the American Sleep Apnea Association (TFAC-AWAKE) organization website, and was promoted through the TFAC's XM radio, word of mouth, and trucking industry press contacts.

Results: A total of 595 individuals completed the survey. Of these, $55.9 \%$ were positive on the Berlin, $78.3 \%$ had either hypertension or obesity, $69.6 \%$ were obese, $47.6 \%$ had a BMI $>33 \mathrm{~kg} / \mathrm{m}^{2}$, and $20.5 \%$ reported falling asleep at stoplights.
Conclusions: Some commercial drivers willingly assess their OSA risk anonymously online, and a majority of those who do so are obese, have positive Berlin screening questionnaires, and would be required to undergo polysomnography if recommendations made to the FMCSA became regulation. In contrast to reported behavior during actual Commercial Driver Medical Examinations physicals, some commercial drivers will report OSA symptoms if it is "safe" to do so. Sleep health professionals need expedient, non-punitive methods to keep commercial motor vehicle drivers healthy and driving and to raise drivers' awareness of the dangers of drowsy driving and unhealthy lifestyles.

Keywords: Commercial drivers, motor vehicle crash, Federal Motor Carrier Safety Administration, obstructive sleep apnea, Berlin Questionnaire

Citation: Smith B; Phillips BA. Truckers drive their own assessment for obstructive sleep apnea: a collaborative approach to online self-assessment for obstructive sleep apnea. J Clin Sleep Med 2011;7(3):241-245.

$\mathrm{O}$ bstructive sleep apnea (OSA) is associated with an increased risk of motor vehicle crash, ${ }^{1-3}$ and effective treatment with continuous positive airway pressure (CPAP) reduces that risk. ${ }^{4}$ Although evaluation of the risk for crash in commercial motor vehicle drivers with OSA has not been extensively evaluated, ${ }^{2}$ it is likely that OSA increases the crash risk for those drivers as well. Commercial motor vehicle crashes carry an increased risk because of the size of the vehicles, the number of passengers, and the types of cargo that they may carry. Indeed, the fatality rate per 100 million vehicle miles travelled was higher for large trucks than for all vehicles in 2005. ${ }^{5}$ Further, commercial motor vehicle drivers appear to be at increased risk for OSA, with some estimates as high as one in four commercial drivers having OSA. ${ }^{6-8}$

The primary mission of the Federal Motor Carrier Safety Administration (FMCSA) of the Department of Transportation (DOT) is to reduce crashes, injuries, and fatalities involving large trucks and buses. The process by which regulations and guidelines are developed is well described. ${ }^{9}$ As part of this process, the FMCSA commissioned an evidence review and convened a Medical Expert Panel, which made recommendations about identification and management of OSA in commercial

\section{BRIEF SUMMARY \\ Current Knowledge/Study Rationale: We completed this study to investigate whether truckers would use an online screening test of their risk for sleep apnea, and to find out the proportion of those who did so that would be likely to have sleep apnea if they underwent formal testing. Study Impact: A majority of truckers who take an online screening test for sleep apnea test positive and are obese. The discordance between the reported symptoms on an anonymous screen and during their Com- mercial Driving Medical Examination physicals is a cause of concern, and suggests that truckers do not feel safe disclosing symptoms of sleep apnea during their formal physical examinations.}

drivers. ${ }^{10}$ Among the recommendations of the Medical Expert Panel were that certain individuals should be required to undergo evaluation to confirm the diagnosis of and to stratify the severity of OSA, including those categorized as high risk for OSA according to the Berlin Questionnaire, ${ }^{11}$ those with a body mass index $(\mathrm{BMI}) \geq 33 \mathrm{~kg} / \mathrm{m}^{2}$, or those judged to be at risk for OSA based on a clinical evaluation. After further review of the evidence and of the Medical Expert Panel's recommendations, the Medical Review Board of the FMCSA recommended adopting the Medical Expert Panel's report and recommendations in 
toto, with the exception that individuals with a BMI $\geq 30 \mathrm{~kg} /$ $\mathrm{m}^{2}$ (rather than $33 \mathrm{~kg} / \mathrm{m}^{2}$ ) should undergo evaluation for OSA. ${ }^{12}$

As of this writing, these recommendations to the FMCSA have not yet been acted on by the FMCSA, but they are in the public domain on the DOT website, and have resulted in considerable discussion and anticipatory change. In particular, commercial drivers themselves are understandably concerned about the impact of these guidelines on their careers.

As a service to its members, the Truckers for a Cause-Alert Well and Keeping Energetic (TFAC-AWAKE) organization of truck drivers sought to develop a truck driver sleep apnea selfevaluation web page to be hosted on the TFAC-AWAKE website, based on the proposed FMCSA guidelines for screening for OSA. Individuals from this organization approached us for assistance with screening tools and with website development to help drivers screen themselves for sleep apnea.

The aims of this study (in addition to establishing a tool that truck drivers could use to assess their risk for OSA) were to determine if commercial drivers would actually employ an online tool to assess risk, to collect demographic data from selfselected commercial drivers, and to make correlations between drowsy driving and risk factors for OSA.

We hypothesized that some truckers would, indeed, use the online tool, and that a large proportion of those who chose to do so would have a high probability for OSA. We hypothesized that those who reported classic OSA symptoms (snoring, sleepiness) would be more likely to report drowsy driving, but that objective data (BMI, hypertension history) would predict sleepy driving better than would subjective data.

\section{METHODS}

\section{Survey Development and Promotion}

The survey instrument was a web-based version of the Berlin Questionnaire, ${ }^{11}$ as recommended by the Medical Expert Panel of the Federal Motor Carrier Safety Administration. The Berlin Questionnaire consists of a series of questions about risk factors for sleep apnea, and is presented in three sections (one about sleepiness, one about snoring, and one about BMI and hypertension). We included an automatic BMI calculator to facilitate that part of the data collection. An individual is considered at high risk if positive on 2 of 3 of the sections. This screening instrument was computerized for use on the TFAC-AWAKE website. The survey was promoted through the TFAC's XM radio, word of mouth, and trucking industry press contacts.

\section{Participants}

Participants could take the survey anonymously from any computer. Potential participants accessed the survey by visiting the TFAC-AWAKE website and logged onto the "Sleep Apnea Checker for Drivers" link (http://awake.truckersforacause. com). Individuals who took the survey were identified only through their computer's IP (Internet Protocol) address; this was done to ensure anonymity and to be sure that each participant completed the survey only once. After completion of the online questionnaire, participants immediately learned whether their Berlin questionnaire score was positive or negative. If their Berlin score was positive, they also received a link to a National Sleep Foundation website list of sleep centers.

\section{Data Collection}

The website link was active for data collection from January 11, 2010 until September 24, 2010. After that point, the link remained active for truckers' continued usage, but it no longer stored data into the data set. This cut-off point for data collection was created because internet service providers may change a user's IP address over time. Some participants may try to "game" the system to manipulate the survey results with multiple completions of the form. The survey window was open for a short time to reduce this threat to the dataset.

\section{Data Analysis}

We applied the R statistical package (http://r-project.org) to construct several logistic regression ${ }^{13}$ models on our dataset. We treated the question "Have you ever nodded off or fallen asleep while driving a vehicle?" as a binary response variable. The Berlin question "Has anyone noticed that you quit breathing during your sleep?" had 4 possible answers, ranging from "Never or nearly never" to "Nearly every day." We transformed this variable to be binary by taking "Never or nearly never" to indicate "No" and any other answer to indicate "Yes." For both response variables (Drowsy Driving and Witnessed Apneas), we generated statistical models using logistic regression and each of the other variables in our study as univariate independent factors.

Hypertension was also treated as a binary variable, based on yes or no answers to the question, "Do you have high blood pressure?" We treated the ordinal answers to questions such as "How often do you snore?" and "How often do you feel tired or fatigued after you sleep" as continuous variables with the lowest answer ("Never or nearly never") as 0 , and the highest answer ("Nearly every day") as 4 . We treated these variables as continuous for the ease of reporting the results, but treating them as ordinal variables yields the same interpretation in our sample.

This study was granted IRB exemption Certification for Protocol No 10-0027-X2B from the University of Kentucky Office of Research Integrity.

\section{RESULTS}

Over the 9 months of data collection, a total 595 individuals took the survey. Of these, $55.9 \%$ scored positive on the overall Berlin survey, and $78.3 \%$ were positive on objective (BMI, hypertension) section. We have no way to determine the non-completion rate of the study. We did not collect data on participants' age. The type and treatment of variables in our data set as well as descriptive statistics can be found in Table $\mathbf{1}$.

\section{Obesity and Hypertension}

More than two-thirds of the respondents were obese: $69.6 \%$ had a BMI $>30 \mathrm{~kg} / \mathrm{m}^{2}$, and $47.6 \%$ had a BMI $>33 \mathrm{~kg} / \mathrm{m}^{2} ; 35.6 \%$ had a BMI $>35 \mathrm{~kg} / \mathrm{m}^{2}$. Figure 1 presents a frequency histogram of BMIs of the respondents. Obesity correlated with snoring loudness (the louder the snoring, the greater likelihood of obe- 
Table 1-Descriptive statistics and variable types

Question or Variables
Height
Weight
BMI
Gender
Overall Berlin Score
Have you ever nodded off or fallen asleep while driving a vehicle?
Do you have high blood pressure?
Do you snore?
Does your snoring bother other people?
How often do you snore?
How often has someone noticed that you quit breathing during your sleep?
How often do you feel tired or fatigued after your sleep?
During your waking time, how often do you feel fatigued, or not up to par?
How loud is your snoring?

Scale Type
Continuous
Continuous
Continuous
Binary
Binary
Binary
Binary
Categorical
Categorical
Ordinal
Ordinal
Ordinal
Ordinal
Ordinal

\section{Mean, Median, or Mode ${ }^{\circ}$}

70.29 in.

$240.10 \mathrm{lbs}$.

$33.94 \mathrm{~kg} / \mathrm{m}^{2}$

$91.8 \%$ Male

$55.9 \%$ Positive

$20.5 \%$ Yes

$39.5 \%$ Yes

$66.2 \%$ Yes

$43.4 \%$ Yes

Median: 1-2 times a week

Median: Never or nearly never

Median: 1-2 times a month

Median: 1-2 times a month

Median: Slightly louder than breathing

†In our logistic regression analyses, ordinal variables were treated as univariate continuous variables ranging from $0-4$, where 0 was the lowest response and 4 was the highest response. ${ }^{\circ}$ The descriptive statistic was chosen based on the scale type of the variable in question. Continuous data, such as GPA or weight, is best described by its mean. Ordinal data, such as great, good, or bad, is best described by its median. Categorical data, such as gender or race, is best described by the mode. ${ }^{19}$

sity) $(\mathrm{p}<0.05)$ and with hypertension $(\mathrm{p}<0.05)$. More than a third (39.5\%) of participants reported hypertension, which correlated with feeling tired/fatigued after sleep $(\mathrm{p}<0.05)$

\section{Sleepy Driving}

With regard to drowsy driving, $20.5 \%$ answered positively to the Berlin Questionnaire query about falling asleep while driving. Correlates of sleepy driving are reported in Table 2. Sleepy driving was not correlated with BMI, hypertension $(\mathrm{p}=0.07)$ or gender, but strongly correlated with severity and frequency of snoring and with witnessed apneas.

\section{Witnessed Apneas}

Twenty-one percent of the commercial motor vehicle drivers endorsed the symptom of witnessed apneas; correlates of this symptom are also presented in Table 2. Witnessed apneas were not correlated with BMI, gender, or hypertension, but correlated with classic OSA symptoms.

\section{DISCUSSION}

The primary findings of this report are that some commercial drivers willingly assess their OSA risk anonymously online, and a majority of those who do so are obese, have positive Berlin screening questionnaires, and would be required to undergo polysomnography (PSG) if suggested guidelines become regulation. Those who reported classic OSA symptoms (snoring, sleepiness) were more likely to report drowsy driving. Contrary to our hypothesis, however, objective data (BMI, hypertension history) did not predict sleepy driving better than subjective data (sleepiness, snoring).

To our knowledge, this report is the first to result from an anonymous screening tool for obstructive sleep apnea in com-
Figure 1-Histogram of body mass indices of 595 selfselected commercial drivers

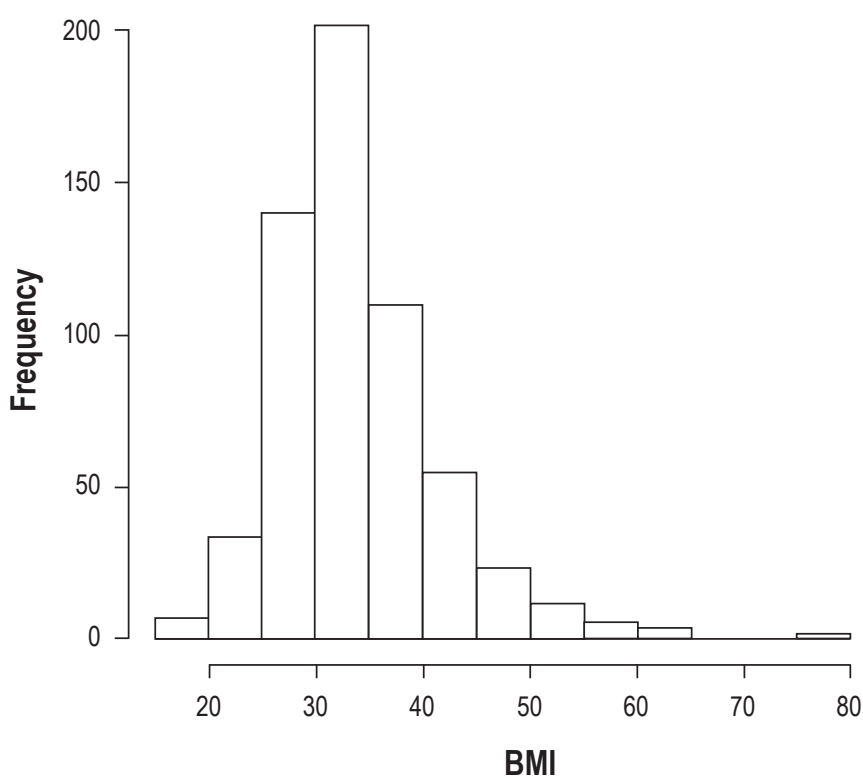

mercial drivers. Nearly 600 individuals took the anonymous screen within a few months. In return, they received fairly specific information about whether or not they would be likely to be referred for polysomnography at their next Commercial Driver Medical Examination, should recommendations currently being considered by the FMCSA become policy. Although this project was initially conceived as a service to commercial drivers, it enabled us to gain some insight into demographics and symptoms in this group. As we hypothesized, those who reported symptoms of sleep apnea (snoring, witnessed apneas) were more likely to report drowsy driving. We were surprised 


\section{Table 2-Correlates of drowsy driving and witnessed apnea Question}

Overall Berlin Score
Do you snore?
How loud is your snoring?
Does your snoring bother other people?
How often do you snore?
How often has someone noticed that you quit breathing during your sleep?
How often do you feel tired or fatigued after your sleep?
During your waking time, how often do you feel fatigued, or not up to par?

\begin{tabular}{cc}
\multicolumn{2}{c}{ Significance (i.e., p-value) ${ }^{*}$} \\
\hline Drowsy Driving & Witnessed Apneas• \\
$<0.0001$ & $<0.0001$ \\
$<0.01$ & $<0.001^{\circ}$ \\
$<0.0001$ & $<0.0001$ \\
$<0.01$ & $<0.01$ \\
$<0.0001$ & $<0.0001$ \\
$<0.0001$ & $\mathrm{n} / \mathrm{a}^{\dagger}$ \\
$<0.0001$ & $<0.0001$ \\
$<0.0001$ & $<0.0001$
\end{tabular}

†This value has been omitted since the test described here would be correlating the variable with itself. "Respondents who answered "yes" had the stated $p$-value, respondents who answered "I don't know" were weakly correlated with $p=0.10478$. "The significance value is taken from a univariate logistic regression model with the questions as univariate independent variables and the 2 responses as the dependent variables. $\cdot$ This variable was transformed such that "Nearly every day" and "3-4 times/week" were a positive response and every other response was a negative response.

to discover that symptoms such as sleepiness and snoring were correlated with drowsy driving more robustly than objective data in this anonymous, online survey. Previous reports ${ }^{14,15}$ have indicated that objective findings (e.g., BMI, blood pressure) were more predictive of sleep apnea in commercial drivers than subjective symptoms. Indeed, in a prospective study of 440 commercial drivers, Parks et al. found that every driver who was subsequently found to have OSA by polysomnography had denied related symptoms on his Commercial Driver Medical Examination, ${ }^{14}$ and Talmage et al. actually found that the Epworth Sleepiness Scale correlated inversely with apnea plus hypopnea index (AHI) as determined by polysomnography in truckers who were undergoing required DOT physicals. ${ }^{15}$ Possibly, drivers understand that reporting symptoms of sleep apnea on their DOT evaluations will result in potentially lengthy and expensive disruptions in work, and thus avoid reporting them on their commercial driver's license certifying exam. However, they can and did report these symptoms on this anonymous web-based screening tool. This result suggests that commercials drivers are sincerely interested in knowing whether they might have sleep apnea.

The prevalence of obesity in this sample is strikingly high, with about $70 \%$ of respondents having a BMI over 30 . This compares with the national average of $26.7 \%$ in $2009 .{ }^{16}$ There are several different recommendations about use of BMI in screening commercial drivers for obstructive sleep apnea. The 2006 Triagency Task Force of the American College of Chest Physicians, National Sleep Foundation, and American College of Occupational and Environmental Medicine ${ }^{2}$ recommended sleep apnea evaluation for drivers who had BMIs over $35 \mathrm{~kg}$ / $\mathrm{m}^{2}$. That cut point would apply to more than a third $(35.6 \%)$ of this sample. The Medical Expert Panel convened by the FMCSA produced conflicting guidance; at one point, it included drivers with BMIs $>33 \mathrm{~kg} / \mathrm{m}^{2}$ among those who should be disqualified immediately or denied certification, but in another section it noted, "Individuals with a $\mathrm{BMI} \geq 33 \mathrm{~kg} / \mathrm{m}^{2}$ may be conditionally certified for one month pending the findings of a sleep study." The panel noted that this period should be less than one week. However, given the current infrastructure for sleep studies in the United States, obtaining a sleep study within one week is unlikely to be feasible in many cases. Consequently, the panel recommended that a transition period of two years be allowed, during which time efforts should be made to improve the infrastructure so that the period between requesting a sleep study and obtaining that study can be reduced to one week for certification purposes." 10 However interpreted, this recommendation would apply to nearly half of this sample. In its deliberations, the Medical Review Board of the FMCSA recommended that drivers with a BMI over 30 be required to undergo testing for sleep apnea prior to certification, ${ }^{16}$ which would affect nearly two-thirds of the individuals who took used our survey.

Strengths of this study include the large number of participants, the specific and select method for recruitment of commercial drivers, and the robust correlation of classic sleep apnea symptoms with drowsy driving. Weaknesses include the fact that data were collected anonymously (we have no way of knowing who the respondents were), and the self-reported and subjective nature of our screening tool. However, the Berlin Questionnaire was recommended by the FMCSA's Medical Expert Panel as an appropriate screening too ${ }^{10}$ and was among the most specific of screening tools for OSA in a web-based model. ${ }^{17}$ Perhaps the biggest weakness is that this self-selected group of respondents included mostly individuals who already suspected that they had sleep apnea. While acknowledging that this is true, we still believe that these survey results present some useful findings.

A significant weakness of this study the fact that the respondents to the survey were self-selected and thus were almost certainly at higher risk for OSA than the general commercial driver population. These results may not be generalizable to the overall commercial driver population, although they may be generalizable to the overweight/obese group of drivers. In addition, because of the need for simplicity and brevity, we did not collect demographic data from survey participants. Further, because of our IRB requirements for anonymity, it is impossible to verify if the individuals who completed the survey were actually commercial vehicle drivers, or to be absolutely certain that each respondent only completed the survey once. 
In an interesting study of clinicians who likely have handson experience with screening for OSA in commercial drivers, Durand and Kales ${ }^{18}$ surveyed American College of Occupational and Environmental Medicine (ACOEM) members regarding recent consensus guidelines for screening commercial drivers for OSA. ${ }^{2}$ Fewer than half of these clinicians used consensus guidelines or another specific protocol to screen for OSA, citing as explanations that they were unaware of guidelines $(36 \%)$, that such guidelines were too complicated $(12 \%)$, that it would affect client retention (10\%) or cause inconvenience to drivers $(10 \%)$. Thus, many commercial driver medical examiners may avoid rigorous screening for OSA because it is complicated or might adversely affect drivers or their practice. In addition, drivers themselves who are suspected of having OSA by their medical examiner and who are referred for screening frequently fail to follow-up with sleep testing or with the examiner who recommended it. ${ }^{14}$ So despite the fact the untreated sleep apnea increases crash risk and has a high prevalence in commercial drivers, many medical examiners are not screening for it, and drivers are denying symptoms on their certifying examinations. As noted by the FMCSA's Medical Expert Panel, cost, complexity, and delay probably contribute an inability to diagnose and expediently treat commercial drivers. Sleep health professionals need expedient, non-punitive tools to keep commercial motor vehicle drivers healthy and driving.

\section{REFERENCES}

1. Tregear S, Reston J, Schoelles K, Phillips B. Obstructive sleep apnea and risk of motor vehicle crash: systematic review and meta-analysis. J Clin Sleep Med 2009;5:573-81.

2. Hartenbaum N, Collop N, Rosen IM, et al. Sleep apnea and commercial motor vehicle operators: Statement from the joint task force of the American College of Chest Physicians, the American College of Occupational and Environmental Medicine, and the National Sleep Foundation. Chest 2006;130:902-5.

3. Ellen RB, Marshall SC, Palayew M, Molnar FJ, et al. Systematic review of motor vehicle crash risk in persons with sleep apnea. J Clin Sleep Med 2006;2:193-200.

4. Tregear S, Reston J, Schoelles K, Phillips B. Continuous positive airway pressure reduces risk of motor vehicle crash among drivers with obstructive sleep apnea; systematic review and meta-analysis. Sleep 2010;33:1373-80.

5. http://www.fmcsa.dot.gov/facts-research/facts-figures/analysis-statistics/cmvfacts.htm

6. Howard ME, Desai AV, Grunstein RR, et al. Sleepiness, sleep-disordered breathing, and accident risk factors in commercial vehicle drivers. Am J Respir Crit Care Med 2004;170:1014-21.

7. Moreno $\mathrm{CR}$, Carvalho FA, Lorenzi $\mathrm{C}$, et al. High risk for obstructive sleep apnea in truck drivers estimated by the Berlin Questionnaire: prevalence and associated factors. Chronobiol Int 2004;21:871-9.
8. Pack Al, Maislin G, Staley B, et al. Impaired performance in commercial drivers: role of sleep apnea and short sleep duration. Am J Respir Crit Care Med 2006:174:446-54

9. http://www.fmcsa.dot.gov/rules-regulations/topics/medical/Overview-FMCSAMedStds-Process.pdf.

10. http://www.fmcsa.dot.gov/rules-regulations/TOPICS/mep/report/Sleep-MEPPanel-Recommendations-508.pdf

11. Netzer NC, Stoohs RA, Netzer CM, Clark K, Strohl KP. Using the Berlin Questionnaire to identify patients at risk for the sleep apnea syndrome. Ann Intern Med 1999;131:485-91.

12. http://www.mrb.fmcsa.dot.gov/documents/Fin_Meet_Min_Jan28_2008MRB_ Meet_Revised11-24-09.pdf

13. Agresti A. Building and applying logistic regression models. An Introduction to categorical data analysis. Wiley: Hoboken, NJ, 2007.

14. Parks P, Durand G, Tsismenakis AJ, Vela-Bueno A, Kales S. Screening for obstructive sleep apnea during commercial driver medical examinations. J Occup Environ Med 2009;51:275-82.

15. Talmage JB, Hudson TB, Hegmann KT, Thiese MS. Consensus criteria for screening commercial drivers for obstructive sleep apnea: evidence of efficacy. J Occup Environ Med 2008;50:324-9

16. http://www.cdc.gov/mmwr/pdf/wk/mm59e0803.pdf

17. Hwang KO, Hamadah AM, Johnson CW, Thomas EJ, Goodrick GK, Bernstam EV. Screening for obstructive sleep apnea on the internet: randomized trial. Am J Med 2009:122:961.e1-6.

18. Durand G, Kales SN. Obstructive sleep apnea screening during commercial driver medical examinations: a survey of ACOEM members. J Occup Environ Med 2009;51:1220-6.

19. Stevens SS. On the theory of scales of measurement. Science 1946;103:677-80.

\section{ACKNOWLEDGMENTS}

Both authors had full access to the data and participated in writing this report. We gratefully acknowledge the help of Robert Stanton and Gary Hull, and Truckers for a Cause Chapter of Alert Well and Keeping Energetic of the American Sleep Apnea Association (TFAC-AWAKE), who initiated this project and hosted the survey on the TFAC-AWAKE website.

\section{SUBMISSION \& CORRESPONDENCE INFORMATION}

Submitted for publication November, 2010

Submitted in final revised form November, 2010

Accepted for publication December, 2010

Address correspondence to: Barbara A Phillips, M.D., M.S.P.H., F.C.C.P., Division

of Pulmonary, Critical Care and Sleep Medicine, University of Kentucky College of Medicine, 800 Rose St, Lexington, KY, 40536-00298; E-mail: rfphil1@uky.edu

\section{DISCLOSURE STATEMENT}

This was not an industry supported study. Dr. Phillips has received honoraria/consulting fees from ResMed, Philips, and Cephalon, and has served on the Medical Advisory Board of the Federal Motor Carrier Safety Administration. The other author has indicted no financial conflicts of interest. 